\title{
Análise do Prêmio APS Forte: iniciativas sobre Áreas Remotas e Vulnerabilidade Social
}

Andrea Borghi Moreira Jacinto, Aline Ludmila de Jesus, Denise Lins de Sousa, Luana Andrade Benício

RESUMO

Este artigo analisa as experiências e iniciativas realizadas em áreas remotas e/ou em cenários de vulnerabilidade social e que visam trazer melhorias à população no âmbito da Atenção Primária à Saúde (APS).

Palavras-chave: Atenção Primária à Saúde; Acesso; Prêmio APS.

\section{ABSTRACT}

This article analyzes the experiences and initiatives carried out in remote areas and / or in social vulnerability scenarios that aim to bring improvements to the population within the scope of Primary Health Care (PHC).

Keywords: Prmary Health Care; Access; PHC Prize.
Revista da Rede APS 2020

Publicada em: 04/09/2020

DOI:10.14295/aps.v2i3.146

Andrea Borghi Moreira Jacinto (Ministério da Saúde, Brasil)

Aline Ludmila de Jesus (Ministério da Saúde, Brasil)

Denise Lins de Sousa

(Ministério da Saúde, Brasil)

Luana Andrade Benício (Ministério da Saúde, Brasil)

Correspondência para:

Andrea Borghi Moreira Jacinto andrea.jacinto@saude.gov.br 


\section{MARCo RefERenCial SOBRE O TEMA}

\section{"ÁREAs ReMOtAs E VULNERABILIDADE \\ SOCIAL"}

Considerando as iniciativas locais, municipais ou regionais enviadas para o prêmio APS Forte para

o SUS: Acesso Universal, este artigo analisa as experiências e iniciativas realizadas em áreas remotas e/ou em cenários de vulnerabilidade social e que visam trazer melhorias à população no âmbito da Atenção Primária à Saúde (APS). A análise tem como referencial os princípios e as diretrizes que alicerçam o Sistema Único de Saúde (SUS) e que são referendados pela Lei $\mathrm{n}^{\circ}$ 8.080, de 19 de setembro de 1990, dentre eles: universalidade, integralidade e igualdade da assistência à saúde. Pauta-se, ainda, na Portaria $\mathrm{n}^{\circ} 2.436$, de 21 de setembro de 2017, que versa sobre a Política Nacional de Atenção Básica e traz o princípio da equidade e as diretrizes de regionalização e hierarquização, territorialização, população adscrita e dentre outros para a operacionalização da APS no âmbito do SUS.

Compreende-se que as experiências inscritas e que lidam com o tema "Áreas Remotas e Vulnerabilidade Social" remetem aos desafios inerentes ao direito à saúde preconizado pela Constituição Federal de 1988, sobretudo no que diz respeito à equidade e ao acesso. As experiências trazem à tona diferentes realidades territoriais, sociais e culturais, bem como contextos de vulnerabilidade, explicitando a complexidade no que diz respeito ao cuidado e ao acesso à saúde nos diversos cenários do país. Nesse sentido, busca-se analisar as relações entre a saúde e seus determinantes sociais e como as iniciativas enfrentam esses desafios, contribuindo para o fortalecimento de políticas de equidade que visam ampliar o acesso à saúde e fortalecer o SUS.

A multiplicidade de contextos e grupos sociais reunidos nas 83 (oitenta e três) experiências cadastradas expressa desafios apresentados a gestores e profissionais de saúde para ofertar o cuidado devido às populações sob sua responsabilidade, e lidar com diferentes condições de vida que influenciam a situação de saúde dessas pessoas. Os determinantes sociais de saúde dizem respeito a fatores sociais, econômicos, culturais, étnico raciais, entre outros, que podem determinar problemas de saúde, exposição a riscos, e também a forma como as pessoas acessam ou não os serviços de saúde e o cuidado a que tem direito (Buss, Pelegrine Filho, 2007).

O papel dos cuidados primários de saúde no conceito ampliado de saúde, apresentado na Conferência de Alma Ata, reforça a relação entre a promoção e a proteção da saúde e condições sociais, econômicas e políticas, bem como a importância do trabalho entre diferentes setores, além da saúde, tendo como meta um nível de saúde assentado no desenvolvimento social, econômico e com justiça social (OMS, 1978; Barros et al, 2016). O enfrentamento das desigualdades em saúde, e de vulnerabilidades sociais que afetam diferentes grupos sociais, tem portanto como horizonte a busca pela equidade em saúde, com a transformação de fatores que sejam evitáveis, injustos e desnecessários (Aith, Scalco, 2015)

No caso do Sistema Único de Saúde, o trabalho pela equidade em saúde está previsto tanto na organização e diretrizes mais amplas do sistema e da organização dos serviço da Rede de Atenção à Saúde na oferta do cuidado - como expresso na Lei 8.080 ou na Política Nacional de Atenção Básica (PNAB) -, quanto em políticas de saúde que reconhecem a vulnerabilidade e necessidades de grupos específicos; $O$ conjunto de experiências inscritas demonstra o trabalho em ambas as direções, articulando a PNAB a outras políticas, como as voltadas à saúde de pessoas privadas de liberdade no sistema prisional, à população em situação de rua, a populações do campo, floresta e águas, à população LGBT, aos ciclos de vida, à saúde das crianças e das mulheres, para citar algumas das experiências representadas no Prêmio APS Forte. Importante citar também a articulação dessas políticas com a Política Nacional de Educação Permanente, que convoca e provoca os sujeitos do quadrilátero da formação - ensino, serviço, gestão e controle social - a refletirem de modo permanente a realidade posta e a buscar soluções criativas para a superação dos 
problemas de saúde e, por conseguinte, qualificar as ações no intuito de aumentar a resolubilidade e a eficiência do sistema de saúde (Ceccim; Feuerwerker, 2004).

\section{ANÁLISE DAS EXPERIÊNCIAS INSCRITAS SOBRE O TEMA “ÁreAs REMOtas E VULNERABILIDADE SOCIAL"}

O tema "áreas remotas e vulnerabilidade social" registrou o cadastro de 83 (oitenta e três experiências), reunindo uma gama variada de questões de saúde pública, relacionadas a diferentes cenários e grupos populacionais no contexto da atenção primária no Brasil.

Em relação aos atributos da APS, cinquenta e quatro (54) experiências trataram mais diretamente de questões relacionadas ao acesso; dez (10) experiências enfocaram questões relativas à integralidade; seis (06), explicitaram a questão da equidade; três (03), destacaram o cuidado centrado na pessoa; três (03), observaram a ordenação da rede; três (3),consideraram o tema da universalidade; três (3), trataram da longitudinalidade do cuidado; e finalmente uma (1) experiência centrou-se na questão da participação da comunidade (Figura 1). Ou seja, em uma análise mais panorâmica, o tema do acesso mostra-se central em cerca de $64 \%$ das experiências relativas à vulnerabilidade e áreas remotas. Em seguida, tiveram destaque os atributos/diretrizes da integralidade e da equidade. Apesar dessa distribuição, os princípios doutrinários do SUS - universalidade, equidade e integralidade - foram transversais e trabalhados em praticamente todas as experiências relatadas.

Figura 1 - Distribuição dos atributos da APS nas experiências relacionadas ao tema "áreas remotas e vulnerabilidade social".

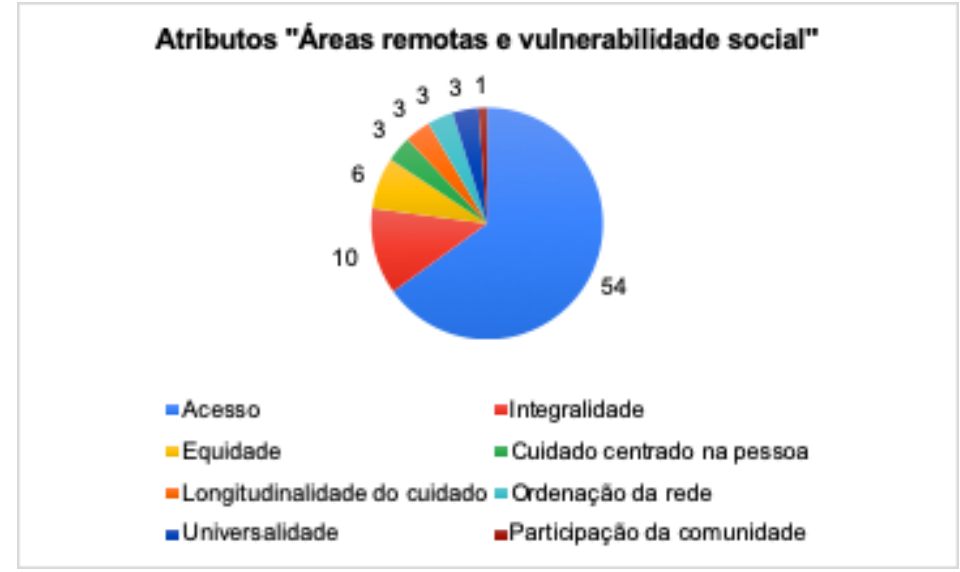

Fonte: Elaboração dos autores.

Do ponto de vista da distribuição das experiências relacionadas a políticas voltadas a populações específicas, algumas políticas estiveram presentes em um número expressivo de experiências. Assim, catorze (14) experiências trataram de temas relacionados à saúde da população do campo, floresta e águas; onze (11) relataram ações no campo da saúde de pessoas privadas de liberdade no sistema prisional; seis (6) relatos deram destaque a saúde da população em situação de rua; cinco
(05) trabalhos deram especial atenção à saúde da população Lésbicas, Gays, Bissexuais, Travestis e Transexuais - LGBT; e cinco (4) experiências trataram de questões relacionadas à saúde indígena.

Em uma análise panorâmica, dezessete (17) experiências não apontaram preliminarmente para grupos ou contextos específicos, mas sim para questões variadas e mais amplas associadas de um modo geral à Política Nacional de Atenção Básica. Nesse conjunto, chamam 
atenção experiências que discutem sobre acesso e serviços itinerantes; processos de trabalho $e$ composição de equipes e redes (indicadores/mapeamento de vulnerabilidade social. Desse conjunto, dois (2) trabalhos referiam-se também a grupos que não envolvem políticas específicas, mas que guardam particularidades e enfrentam graves situações de vulnerabilidade social, como refugiados e catadores de material reciclável.

Além desses temas que registraram maior freqûencia, uma série de experiências apontam o amplo espectro e transversalidade do tema da vulnerabilidade social e de áreas remotas, entrelaçando inúmeras outras políticas, como: Política Nacional de Redução da Morbimortalidade por Acidentes e Violência; Política Nacional de Saúde Bucal; Política Nacional de Atenção à Saúde Auditiva; Política de Atenção Integral à Saúde de Adolescentes em Conflito com a Lei; Política Nacional de Cuidado Paliativo para o SUS; Política Nacional de Saúde da Pessoa com Deficiência; Política Nacional de Educação Permanente em Saúde; Política Nacional de Atenção Integral às Pessoas com Doença Falciforme e outras Hemoglobinopatias; Política Nacional de Práticas Integrativas e Complementares; Política Nacional de Atenção Integral à Saúde da Mulher; Política Nacional de Alimentação e Nutrição; Política Nacional de Atenção Hospitalar. Além das políticas, também programas específicos foram considerados, como: Programa Saúde na Escola; Programa de Residência de Medicina de Família e Comunidade; Programa Melhor em Casa; e Programa Nacional de Imunização.

Feitas essas considerações amplas sobre o conjunto de experiências cadastradas, a análise seguinte tratará de alguns subtemas que se destacaram.

\section{DISCUSSÃO: ANÁLISE COMPARATIVA DAS EXPERIÊNCIAS EM RELAÇÃO AO MARCO TEÓRICO ESCOLHIDO.}

A maior parte das experiências relatadas com a temática áreas remotas ou populações em situação de vulnerabilidade social buscaram promover o acesso de comunidades aos serviços de saúde. O território de atuação das Unidades Básicas de Saúde (UBS) e das equipes da Estratégia de Saúde da Família (eSF), principais portas de entrada da Atenção Primária à Saúde (APS), é um espaço vivo e heterogêneo. Apesar das particularidades que caracterizam cada região, a dinâmica social traz desafios que não escapam ao olhar sensível dos profissionais de saúde, que também trabalham para promover transformações na realidade: a chegada de ocupações, imigrantes e refugiados, grupos em situação de rua, catadores de materiais recicláveis, profissionais do sexo, adolescentes cumprindo medidas socioeducativas, população carcerária, ribeirinhos, indígenas, ciganos, LGBTs, trabalhadores expostos a agrotóxicos, dentre outros perfis de usuários que enfrentam dificuldades para acessar os serviços de saúde.

Como forma de alcançar populações historicamente excluídas e diminuir a distância entre elas e os serviços de saúde, a estratégia mais utilizada foram os atendimentos itinerantes: unidades de saúde móveis (UBS fluviais, consultórios na rua, ônibus adaptados, carretas ou pontos de apoio nos espaços disponíveis nas comunidades etc.) e ações de saúde que vão às comunidades mais distantes da UBS, muitas vezes com horários alternativos para pessoas que trabalham durante o dia. A descentralização dos serviços, com a oferta de atendimento além dos muros da UBS, foi fundamental para garantir o direito à saúde das populações mais vulneráveis, além de fortalecer o vínculo com a comunidade. Em relação às UBS fluviais e ESF ribeirinhas, os relatos afirmam que o financiamento diferenciado do governo federal e estadual é fundamental na promoção, por parte dos municípios, de ações de inclusão das populações específicas e efetivação dos serviços de saúde nos territórios ribeirinhos.

Algumas experiências proporcionaram atendimento de atenção especializada dentro da estrutura da APS, outras viram na articulação intersetorial uma oportunidade de ofertar um cuidado integral, além de dar maior resolutividade às demandas e qualificar o trabalho dos profissionais. Houve relatos de interlocução com a escola enquanto espaço de promoção da saúde. Parcerias com 
universidades, por exemplo, por meio de residências médicas e integração entre ensino e serviço, se mostraram uma alternativa à falta de profissionais.

Dentro desse horizonte, é interessante observar comparativamente alguns campos específicos de atuação. Assim, segue-se uma descrição mais detalhada sobre experiências relacionadas a saúde de pessoas privadas de liberdade no sistema prisional; da população em situação de rua; de práticas de educação permanente; de saúde da mulher em situação de vulnerabilidade, e finalmente, sobre as experiências relativas a populações do campo, floresta e águas.

Diversas experiências pautaram questões referentes ao direito à saúde dentro dos sistemas penitenciários, a partir da análise sobre como a Atenção Básica está presente dentro desses espaços. As iniciativas lograram em desafiar a efetivação dos princípios referendados pela Lei $\mathrm{n}^{\circ} 8.080$, de 19 de setembro de 1990 - de universalização, equidade e integralidade em ambientes que vulnerabilizam a saúde individual e coletiva, endossando o preceito de que "a saúde é direito de todos e dever do Estado". Os indivíduos que se encontram em sistemas prisionais estão privados da liberdade, no entanto, devem ter os direitos sociais e humanos resguardados, incluindo o direito à saúde - física e mental. 0 sistema prisional brasileiro tem como características a superlotação, a insalubridade, a precariedade das condições de moradia e alimentação, bem como a dificuldade de acesso a medicamentos e atendimentos médicos. Esses fatores propiciam a propagação de enfermidades, infecções e corroboram o agravamento de transtornos mentais. 0 ambiente carcerário possui altas taxas de prevalência de doenças infecciosas e crônicas, o que exige ações de promoção, prevenção e recuperação da saúde, de forma longitudinal e integral. As iniciativas defrontaram esses desafios e propuseram estratégias para a efetivação de ações de promoção, prevenção e reabilitação da saúde voltadas às pessoas privadas de liberdade, à luz dos princípios do SUS, dos atributos essenciais e derivados da Atenção Primária e da Política Nacional de
Atenção à Saúde das Pessoas Privadas de Liberdade no Sistema Prisional (PNAISP).

Uma das estratégias apontadas em algumas experiências foi a realização de reuniões com as equipes de Saúde da Família que atuam em sistemas penitenciários, a fim de analisar os principais pontos de vulnerabilidade da população carcerária e, a partir disso, efetivar ações como: testes rápidos, aferição da pressão arterial, tratamentos específicos para escabioses e transtornos mentais. Destaca-se também a estratégia adotada de elaboração de cronograma de visitas de uma equipe multidisciplinar nas unidades prisionais; inclusive experiências relataram que conseguiram espaço próprio para atendimento dentro desses espaços depois de um tempo, fortalecendo a longitudinalidade e a integralidade dos serviços de acesso à saúde.

Outra estratégia apontada em uma iniciativa foi uma ação realizada com um infectologista que fazia visitas no presídio, a fim de realizar testes e analisar a carga viral dos (as) reeducandos (as). Os pacientes que foram diagnosticados com HIV, e que desejaram tratamento, aderiram à Terapia Antirretroviral (TARV). Essa experiência adaptou o cuidado especializado à Unidade Básica em Saúde. Ainda sobre ações específicas de cuidado à saúde, uma Universidade elaborou um projeto de ensino-aprendizagem através do qual iniciaram intervenção em um sistema prisional feminino. Esse projeto propiciou a realização de exames ginecológicos, no intuito de investigar e detectar alterações e doenças no colo do útero.

As questões referentes ao direito à saúde também foram evidenciadas nas experiências sobre o acesso à saúde da população em situação de rua. As iniciativas buscaram a promoção do acesso à saúde através do estabelecimento de vínculo e da desconstrução do estigma em relação a essa população. Ressalta-se que a invisibilidade e estigmatização são problemas que reverberam na população de rua e, consequentemente, na sua saúde; muitos deixam de buscar atendimento médico dada a dificuldade de encontrar acolhimento. Estabelecer vínculos entre a população em 
situação de rua é os serviços de saúde é, portanto, um dos grandes desafios.

Nesse sentido, as iniciativas trabalharam com a importância de sensibilizar profissionais de saúde, a fim de aumentar o atendimento humanizado e a escuta qualificada, fortalecendo o vínculo dessa população com os serviços de saúde. Inclusive, em uma das experiências, utilizou-se a estratégia de entregar "kits de saúde" com líquidos e alimentos, com objetivo de estabilizar a glicemia e diminuir a ansiedade das pessoas em situação de rua. Essa ação possibilitou a aceitabilidade em relação ao cuidado em atenção primária, fortalecendo o vínculo com os serviços de saúde. Outras ações, acoplaram ações culturais, como o teatro de rua, às ações de saúde, trabalhando as competências culturais e fortalecendo os princípios de equidade, universalidade e integralidade, ao mesmo tempo em que se buscava resgatar a dignidade das pessoas que se encontram nesse quadro de vulnerabilidade social.

Outras experiências foram realizadas em locais de ocupações e de vulnerabilidade social, com o intuito de levar o cuidado à saúde à população. Essas experiências têm como elemento comum o extrapolamento das ações no âmbito das Unidades Básicas, potencializando novas formas de acesso, cuidado e promoção da saúde.

Algumas experiências objetivaram retratar a importância de promover práticas de educação permanente, no intuito de qualificar os serviços de saúde na melhoria do cuidado e ampliação do acesso da população à saúde pública, principalmente em áreas com baixa cobertura de atenção primária devido, sobretudo, à dificuldade de alocar profissionais médicos em áreas distantes dos grandes centros urbanos. 0 Programa de Residência de Medicina de Família e Comunidade tem permitido a ampliação do acesso ao SUS, em especial às consultas médicas, e a adesão de pelos menos dois anos do profissional, com produção de vínculos comunitários e um processo formativo que entende e atende a necessidade das pessoas, suas famílias e comunidades. A experiência de articulação ensino-serviço aumentou a capacidade de oferta de ações e atividades de cuidado, especialmente nas áreas mais remotas e de vulnerabilidade social, potencializando o acesso dos usuários e o fortalecimento da APS, na perspectiva de ampliar o número de profissionais com competências profissionais para atuação em Unidade Básicas de Saúde, em conformidade com os atributos da APS.

Experiências de boas práticas para a garantia da universalidade, promoção do acesso e cuidado integral da atenção à saúde de mulheres em situação de vulnerabilidade também se destacaram nos relatos apresentados. As experiências tiveram como público-alvo mulheres de baixa renda, população carcerária feminina e indígena, e trouxeram relatos que demonstram que a melhora na cobertura de ações voltadas a mulher, como prevenção e proteção contra o câncer de colo de útero, é uma das principais ações no nível primário para diminuir a incidência e mortalidade da população feminina. Essas medidas compreendem uma parte das ações prioritárias do plano de ação global para a prevenção e controle de doenças crônicas não transmissíveis de 2013-2020.

Finalmente, cabem algumas observações a respeito das catorze experiências relacionadas a populações do campo, floresta e águas, ou de modo mais geral, a áreas remotas e rurais. São relatos de vários pontos do país, descrevendo territórios, profissionais e grupos no Amazonas, Pará, Rio Grande do Sul, Minas Gerais, Paraná, Rio de Janeiro, Santa Catarina. As iniciativas descritas têm diferentes protagonistas secretarias municipais e estaduais de saúde; unidades de saúde; profissionais de equipes de saúde da família; escolas médicas. Porém, entre todas elas e as diferenças que trazem territórios recortados por estradas de chão, furos e igarapés, assentamentos rurais, quilombos, barrancos de rio, plantações de soja ou montanhas - a grande questão que atravessa as experiências é sobre acesso e acessibilidade. E nessa direção, mostrou-se predominante nos relatos a utilização de estratégias móveis e itinerantes para a ampliação do acesso e qualificação do cuidado, e a notícia dos bons resultados produzidos por elas, conforme relatos de municípios como Itacoatira e Coar (AM)i, Barcarena (PA), Quissânia (RJ), Aceguá (RS), para citar alguns. 
As experiências chamaram atenção também à importância da articulação entre setores da saúde e trabalhos intersetoriais. Assim, por exemplo, do Paraná descreve-se a implantação do Plano Estadual de Mitigação e Atenção à Saúde da População Exposta a Agrotóxicos, que articula de modo sistemático ações da vigilância e da atenção primária, apoiando municípios e capacitando profissionais, como os ACS, a incluírem em suas rotinas ações de prevenção, atenção e monitoramento. Ou o relato sobre como o internato rural em cursos de medicina pode ampliar o acesso e o vínculo com populações rurais, por meio da articulação entre municípios e Instituições de Ensino Superior - o que também contribui para a formação e fixação de profissionais em áreas remotas. A importância da articulação intra e intersetorial no trabalho das equipes é trazida ainda nas duas experiências relativas à saúde bucal junto a populações rurais no Maranhão e no Amazonas; e vários dos trabalhos mencionam também a importância do apoio de escolas, igrejas, associações comunitárias, inclusive para garantir espaço e recursos compartilhados ao atendimento nas comunidades e territórios. De um modo geral, "estar no território" é descrito não só como estratégia efetiva de levar os serviços e o cuidado às populações e grupos em áreas rurais e remotas, como nos relatos de Ladainha (MG), ou Rio das Antas (SC). Levar o cuidado e promover a saúde junto às comunidades rurais, em várias experiências, é algo apontado como forma de qualificar equipes - "transformação pessoal e profissional" ampliando a compreensão dos profissionais sobre as vulnerabilidades, necessidades e desafios vividos cotidianamente pelas pessoas nessas regiões, promovendo conhecimentos mútuos, vínculo e respeito; fortalecendo a competência cultural das equipes. Por fim, interessante e recorrente em várias experiências é a utilização de mapeamentos e diagnósticos pelas equipes de saúde da família para o planejamento do trabalho, em diálogo e interação com as comunidades e as dinâmicas

1 Wakerman, J., Humphreys, J.S., Wells, R. et al. Primary health care delivery models in rural and remote Australia - a systematic review. BMC Health Serv Res 8, 276 (2008). dos territórios - que podem variar segundo o tempo da colheita, o calendário religioso, ou o regime dos rios.

De modo geral, os relatos apresentados trouxeram experiências de boas práticas ou inovadoras em resposta às necessidades e à complexidade dos territórios, buscando formas de enfrentar o desafio de incluir populações específicas e vulneráveis. Tais ações tiveram impacto positivo na melhoria dos indicadores e condições de saúde das populações. Nesse aspecto, vale todavia, observar um dado curioso: do conjunto das 83 experiências cadastradas, somente 04 foram descritas como "inovadoras". O restante, 79 delas, foram classificadas como "boas práticas". O que esse dado sugere? Uma pista talvez possa ser dada por um estudo do outro lado do mundo, sobre saúde de populações rurais e remotas na Austrália, país que reúne um acúmulo importante sobre o tema ${ }^{1}$. Nesta revisão sistemática, cuja motivação foi a necessidade de melhorar resultados ruins da saúde em comunidades rurais e remotas na Austrália, pela melhoria de acesso aos serviços de saúde, chega-se à seguinte conclusão: ao invés de se buscar cada vez mais inovação, o progresso na melhoria da saúde rural e remota pode estar mais vinculado à consideração do conhecimento adquirido pelos serviços desde os anos 90 , e ao aprimoramento do acesso aos serviços por meio da implementação mais ampla de modelos que demonstram ser bem sucedidos.

\section{CONSIDERAÇÕES FINAIS}

As desigualdades no estado de saúde estão muito associadas à organização social, tendendo a espelhar o grau de iniquidade em cada sociedade. Como o Brasil é um dos países mais desiguais do mundo e devido sua extensão territorial, não são incomuns áreas e populações em situação de vulnerabilidade social, além de populações vivendo em áreas remotas e de 
difícil acesso aos serviços de saúde. A vulnerabilidade social pode provocar estigmas sociais que acabam por determinar um acolhimento precário e consequentemente no distanciamento de usuários aos serviços de saúde mesmo frente as suas necessidades. Por outro lado, a burocratização dos serviços pode dificultar $o$ acesso, principalmente para aquelas populações que vivem em áreas remotas. Estes distanciamentos demandam maiores esforços para implementação de políticas públicas de saúde.

As experiências apontam exemplos de boas práticas que visavam a eliminação das desigualdades em saúde, tendo como norte os princípios do SUS de acesso universal, equidade e integralidade: de um SUS de todos e para todos. Foram relatos de superação de diferenças, como orientação sexual, identidade de gênero, posição socioeconômica, educação, condições de saúde estigmatizadas e situações de marginalização na sociedade, que potencializam a busca por estratégias de inclusão e de reconhecimento do direito a acesso, conforme posto na Constituição Federal de 1988 e o segundo conceito ampliado de Saude da OMS.

Entende-se dessa forma que as experiências relatadas toma como objeto os problemas e as necessidades de saúde da população e incorpora no seu processo de trabalho com vistas à produção de mudanças neste contexto de vulnerabilidades, orientando-se para a melhoria do acesso, da qualidade e humanização na prestação de serviços.

As experiências também mostraram como os serviços da APS são demandados sobre questões que vão além do setor saúde, daí a importância da ação interdisciplinar, em conjunto com a assistência social e outros órgãos, para grupos em situação de vulnerabilidade, como a população em situação de rua. $O$ fortalecimento da rede de proteção social dos vários grupos em vulnerabilidade, como as experiências apontam, contribui para a continuidade do cuidado em saúde e, muitas vezes, transforma as condições de vida da comunidade, possibilitando o acesso a outros serviços públicos.

As experiências inscritas no Prêmio APS Forte apresentam várias estratégias que mostraram avanços e efetividade na ampliação e qualificação do acesso à saúde entre populações vivendo em áreas remotas e em situação de vulnerabilidade social no Brasil. $O$ grande desafio, se levarmos em conta a sugestão australiana, talvez seja portanto o de como se potencializar e multiplicar as lições desenvolvidas nos últimos anos por equipes e profissionais do SUS. 


\section{NOTAS E REFERÊNCIAS}

Ceccim, R. B.; Feuerwerker, L. C. M. O Quadrilátero da Formação para a Área da Saúde: Ensino, Gestão, Atenção e Controle Social. PHYSIS: Revista de Saúde Coletiva, v. 14, n. 1, p. 41- 65, 2004.

BUSS, P. M.; PELLEGRINI FILHO, A. A Saúde e seus Determinantes Sociais. Saúde Coletiva, Rio de Janeiro, v. 1, n. 17, p.77-93, mar. 2007. Disponível em: http://cmdss2011.org/site/wpcontent/uploads/2011/07/A-Sa\%C3\%BAde-e-seus-Determinantes-Sociais.pdf. Acesso em 29/03/20

Aith, F.; Scalco; N. Direito à saúde de pessoas em condição de vulnerabilidade em centros urbanos. Revista USP • São Paulo • n. 107 • p. 43-54 • outubro/novembro/dezembro 2015. Disponível em: http://www.revistas.usp.br/revusp/article/view/115112. Acesso em 27/03/20

Barros, F. P. C; Lopes, J.S; Mendonça, A.V. M.; Sousa, M. F. Acesso e equidade nos serviços de saúde: uma revisão estruturada. Saúde Debate. Rio de Janeiro, v. 40, n. 110, p. 264-271 Jul-set 2016. Disponível em: http://www.scielo.br/pdf/sdeb/v40n110/0103-1104-sdeb-40-110-0264.pdf (acesso 27/03/20). DOI: 10.1590/0103-1104201611020.

Wakerman, J., Humphreys, J.S., Wells, R. et al. Primary health care delivery models in rural and remote Australia - a systematic review. BMC Health Serv Res 8, 276 (2008).

Organização Mundial de Saúde - OMS. Declaração de Alma Ata - 1978. Disponível em: https://opas.org.br/declaracao-de-alma-ata/ 